\title{
Probing the Higgs portal at the LHC through resonant di-Higgs production
}

\author{
Jose M. No ${ }^{1}$ and Michael Ramsey-Musolf ${ }^{2,3}$ \\ ${ }^{1}$ Department of Physics and Astronomy, University of Sussex, BN1 9QH Brighton, United Kingdom \\ ${ }^{2}$ Amherst Center for Fundamental Interactions Department of Physics, \\ University of Massachusetts Amherst, Amherst, Massachusetts 01003, USA \\ ${ }^{3}$ Kellogg Radiation Laboratory, California Institute of Technology, Pasadena, California 91125, USA
}

(Received 4 December 2013; published 27 May 2014)

\begin{abstract}
We investigate resonant di-Higgs production as a means of probing extended scalar sectors that include a $125 \mathrm{GeV}$ Standard Model-like Higgs boson. For concreteness, we consider a gauge singlet Higgs portal scenario leading to two mixed doublet-singlet states, $h_{1,2}$. For $m_{h_{2}}>2 m_{h_{1}}$, the resonant di-Higgs production process $p p \rightarrow h_{2} \rightarrow h_{1} h_{1}$ will lead to final states associated with the decaying pair of Standard Model-like Higgs scalars. We focus on $h_{2}$ production via gluon fusion and on the $b \bar{b} \tau^{+} \tau^{-}$final state. We find that discovery of the $h_{2}$ at the LHC may be achieved with $\lesssim 100 \mathrm{fb}^{-1}$ of integrated luminosity for benchmark parameter choices relevant to cosmology. Our analysis directly maps onto the decoupling limit of the next-to-minimal supersymmetric Standard Model and more generically onto extensions of the Standard Model Higgs sector in which a heavy scalar produced through gluon-fusion decays to a pair of Standard Model-like Higgs bosons.
\end{abstract}

DOI: 10.1103/PhysRevD.89.095031

PACS numbers: 12.60.Fr, 14.80.Ec

\section{INTRODUCTION}

Both ATLAS and CMS observe a Standard Model-like Higgs boson with $\sim 125 \mathrm{GeV}$ mass. While ongoing analyses show that the properties of the newly discovered particle are close to those expected for the Standard Model (SM) Higgs boson $h$, the full structure of the scalar sector responsible for electroweak (EW) symmetry breaking remains to be determined. It is particularly interesting to ascertain whether the scalar sector consists of only one $\mathrm{SU}(2)_{L}$ doublet $(H)$ or if it has a richer structure containing additional states. Addressing this question is an important task for future studies at the Large Hadron Collider.

An interesting avenue for the observation of additional scalar states $X$ occurs in Higgs portal scenarios that contain operators of the form $X H^{\dagger} H$ and $X^{2} H^{\dagger} H$. For $m_{X}>2 m_{h}$, these operators enable the process $p p \rightarrow X^{0} \rightarrow h h$, where $X^{0}$ is the neutral component of $X$, if $X$ is not inert with respect to the Standard Model. Signatures of such resonant di-Higgs production are multiparticle final states comprised of the conventional Higgs boson decay products. Di-Higgs production also occurs purely within the SM, though it cannot receive any enhancement due to an intermediate resonance (for studies of Higgs self-coupling probes with di-Higgs production at the LHC, see Refs. [1-8]).

Higgs portal scenarios are strongly motivated by cosmology. In the presence of a discrete $Z_{2}$ symmetry, $X^{0}$ may be a dark matter candidate. In this case, the cubic operator is forbidden, the vacuum expectation value (vev) of $X^{0}$ vanishes, and resonant di-Higgs production cannot occur. In the absence of a $Z_{2}$ symmetry, however, both the cubic operator and a nonvanishing $X^{0}$ vev can exist. Under these conditions, the presence of the $X^{0}$ may facilitate a strong first-order electroweak phase transition (EWPT) as required by electroweak baryogenesis (for a recent review, see Ref. [9]). In this case, one would encounter a pair of neutral mass eigenstates $h_{1,2}$ formed from mixtures of the two neutral scalar fields, and for $m_{h_{2}} \geq 2 m_{h_{1}}$ resonant di-Higgs production could proceed (see also Ref. [10]).

In what follows, we investigate the prospects for observing such Higgs portal-mediated resonant di-Higgs production in the context of the simplest extension of the SM scalar sector involving one real gauge singlet, $S$. This "xSM" scenario can give rise to a strong first-order EWPT as needed for electroweak baryogenesis in regions of parameter space that would also enable resonant diHiggs production [11,12]. While these and other previous studies have delineated possible signatures of the XSM in the EWPT-favorable regions of parameter space, until now the feasibility of the discovery under these conditions had not been assessed in detail. To our knowledge, what we report below are results of the first detailed collider study of resonant di-Higgs production as it pertains to the possibility of a first-order EWPT. We find that discovery, indeed, appears possible via the $b \bar{b} \tau^{+} \tau^{-}$channel. Given that this final state is somewhat challenging, we are encouraged that prospects for observation of the xSM through other channels such as $b \bar{b} \gamma \gamma$ may also prove feasible.

Apart from the connection to cosmology, study of the xSM also allows for a relatively general analysis of Higgs portal-mediated resonant di-Higgs production. In particular, as we discuss in more detail in Sec. II, the present analysis can be mapped directly onto the "decoupling limit" $m_{A} \gg v$ of the next-to-minimal supersymmetric Standard Model (NMSSM) [13,14] as well as onto other 
scenarios that include additional degrees of freedom not directly relevant to di-Higgs production.

In this study, we concentrate on the $b \bar{b} \tau^{+} \tau^{-}$final state, motivated in part by the analogous work on SM-only nonresonant di-Higgs production as well as by the considerations discussed in Sec. IV. ${ }^{1}$ We find that with an appropriate strategy for background reduction, the discovery of $h_{2}$ at the LHC may be feasible with $\sim 50-100 \mathrm{fb}^{-1}$. Other final states resulting from combinations of Higgs decay products may also provide promising probes of the Higgs portal through resonant di-Higgs production, and we defer an analysis of these possibilities to future work. ${ }^{2}$

The discussion of our analysis leading to this conclusion is organized as follows. In Sec. II we review the theoretical framework and motivation for the xSM. Section III gives the present LHC constraints and discusses other phenomenological considerations. In Sec. IV we discuss the details and present the results of our LHC simulations and analysis, while in Sec. V we discuss their implications.

\section{SINGLET SCALARS BEYOND THE SM}

Singlet scalar extensions of the SM are both strongly motivated and widely studied [16]. In the present instance, we rely on the simplest version as a paradigm for Higgs portal interactions and the prospects for novel collider signatures. At the same time, singlet extensions of the scalar sector are interesting in their own right. From a model-building perspective, singlet scalars arise in various SM extensions, such as those containing one or more additional $U(1)$ groups that occur in string constructions or variants on the NMSSM. Cosmology provides additional motivation. As noted above, the presence of the singlet scalar can enable a strongly first-order EWPT as needed for electroweak baryogenesis, while imposing a $Z_{2}$ symmetry on the potential allows the singlet scalar to be a viable dark matter candidate (for early references, see, e.g. Refs. [17,18]). In principle, one may achieve both a viable dark matter candidate and a strongly first-order EWPT for a complex scalar singlet extension in the presence of a spontaneously and softly broken global U(1) $[19,20]$.

In what follows, we concentrate on the real singlet, though many of the features discussed below will apply to the real component of the complex singlet case as well. The corresponding scalar potential for the SM Higgs doublet $H$ and a real singlet scalar field $S$ is

\footnotetext{
${ }^{1}$ We thank B. Brau for suggesting the study of this final state to us.

${ }^{2}$ As this paper was being prepared for submission, an investigation of these other states appeared in Ref. [15]. The results of the latter analysis differ considerably from ours, as we discuss below.
}

$$
\begin{aligned}
V(H, S)= & -\mu^{2}|H|^{2}+\lambda|H|^{4}+\frac{b_{2}}{2} S^{2}+\frac{b_{4}}{4} S^{4} \\
& +\frac{a_{2}}{2} S^{2}|H|^{2}+\frac{a_{1}}{2} S|H|^{2}+\frac{b_{3}}{3} S^{3}-\frac{a_{1} v^{2}}{4} S .
\end{aligned}
$$

We note that the scalar potential of the NMSSM in the decoupling regime $m_{A} \gg v$ is of the form (1) $[13,14]$. In this limit, the minimal supersymmetric SM Higgs dynamics approaches that of the SM, so that the Higgs dynamics for the NMSSM essentially reduces to that of the SM Higgs coupled to a scalar singlet. Then, our analysis for the xSM could be directly mapped onto that interesting scenario (recent global fits of LHC data in the context of supersymmetric models tend to favor this regime [21-23]). Studies of resonant di-Higgs production in the NMSSM have been carried out [24-26], though in a different context from the present one (e.g. Ref. [24] considered the process $h_{2} \rightarrow h_{1} h_{1}$, with the state $h_{2}$ being the $125 \mathrm{GeV}$ SM-like Higgs scalar).

Following Ref. [27], we have incorporated the last term in Eq. (1) in order to cancel the singlet tadpole generated once the EW symmetry is spontaneously broken, with

$$
H=\frac{1}{\sqrt{2}}\left(\begin{array}{c}
0 \\
h+v
\end{array}\right)
$$

in the unitary gauge and with $v=246 \mathrm{GeV}$. Denoting the neutral component of $H$ by $H^{0}$, the minimization conditions $\partial V / \partial H^{0}=0$ and $\partial V / \partial S=0$ lead to

$H^{0}\left[-2 \mu^{2}+4 \lambda\left(H^{0}\right)^{2}+a_{2} S^{2}+a_{1} S\right]=0$

$S\left[b_{2}+b_{3} S+b_{4} S^{2}+a_{2}\left(H^{0}\right)^{2}\right]=\frac{a_{1}}{2}\left[v^{2} / 2-\left(H^{0}\right)^{2}\right]$.

For positive $b_{2-4}$ and $a_{2}, H$ as given in Eq. (2), and $\lambda v^{2}=\mu^{2}$ as in the Standard Model, the scalar singlet does not develop a zero-temperature vev. ${ }^{3}$ The resulting mass term in the potential is

$$
V_{\text {mass }}=\frac{1}{2}\left(\begin{array}{ll}
h & S
\end{array}\right)\left(\begin{array}{cc}
\lambda v^{2} & a_{1} v / 2 \\
a_{1} v / 2 & b_{2}+a_{2} v^{2} / 2
\end{array}\right)\left(\begin{array}{l}
h \\
S
\end{array}\right) .
$$

The states $h$ and $S$ will mix after EW symmetry breaking if $a_{1} \neq 0$, with the mixing angle denoted by $\theta$. The mass eigenstates $h_{1,2}$ can be expressed in terms of $h$ and $S$ as

$$
\left(\begin{array}{l}
h_{1} \\
h_{2}
\end{array}\right)=\left(\begin{array}{cc}
c_{\theta} & s_{\theta} \\
-s_{\theta} & c_{\theta}
\end{array}\right)\left(\begin{array}{l}
h \\
S
\end{array}\right),
$$

where $c_{\theta} \equiv \cos \theta$ and $s_{\theta} \equiv \sin \theta$ with

\footnotetext{
${ }^{3}$ Note that in Ref. [11], the finite-temperature analysis was performed for a potential not having the linear term in $S$; mapping from one case to the other amounts to performing a linear shift in the field $S$ at zero temperature.
} 


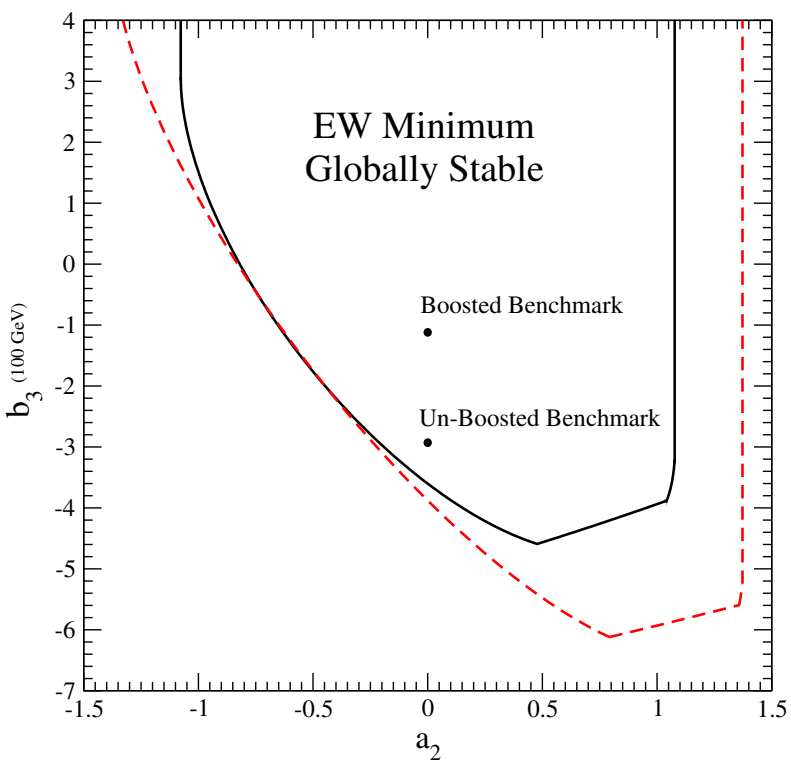

FIG. 1 (color online). Absolute stability region for the EW vacuum in the $a_{2}-b_{3}$ plane for $b_{4}=1, c_{\theta}=0.812404$ and $m_{2}=270 \mathrm{GeV}$ (solid black), $m_{2}=370 \mathrm{GeV}$ (dashed red). The black dots correspond to the benchmark scenarios used in the analysis (see Sec. IV).

$$
\tan \theta=\frac{x}{1+\sqrt{1+x^{2}}}
$$

and

$$
x=\frac{a_{1} v}{\left(\lambda-a_{2} / 2\right) v^{2}-b_{2}} .
$$

The corresponding masses are

$$
\begin{aligned}
m_{ \pm}^{2}= & \frac{1}{2}\left[\left(\lambda+a_{2} / 2\right) v^{2}+b_{2}\right] \\
& \pm \frac{1}{2}\left|\left(\lambda-a_{2} / 2\right) v^{2}-b_{2}\right| \sqrt{1+x^{2}}
\end{aligned}
$$

with $m_{2}=m_{+}$and $m_{1}=m_{-}$.

The scalar potential (1) may then be written in terms of the following seven independent parameters: the two scalar masses $m_{1,2}$; the mixing angle $\theta ; v, a_{2}, b_{3}$ and $b_{4}$. Henceforth, we assume that $h_{1}$ is the Higgs-like state currently being observed at the LHC, with $m_{1}=125 \mathrm{GeV}$, and $h_{2}$ is a heavier scalar state with $m_{2}>2 m_{1}$. The quartic coupling $b_{4}>0$ is needed to assure stability of the potential along the $S$ direction. The value of the effective trilinear $h_{2} h_{1} h_{1}$ coupling

$$
\begin{aligned}
\lambda_{211}= & b_{3} s_{\theta}^{2} c_{\theta}+a_{2} v s_{\theta}\left(c_{\theta}^{2}-s_{\theta}^{2} / 2\right) \\
& +\frac{a_{1}}{4} c_{\theta}\left(c_{\theta}^{2}-2 s_{\theta}^{2}\right)-3 \lambda v c_{\theta}^{2} s_{\theta}
\end{aligned}
$$

is clearly of vital importance to our analysis, since it controls the $\operatorname{Br}\left(h_{2} \rightarrow h_{1} h_{1}\right)$ (see Sec. IV). Note that $\lambda$ and $a_{1}$ are implicitly functions of $m_{1,2}, \theta, v$ and $a_{2}$ via Eqs. (6)-(8).

Considerations of the vacuum structure of the potential introduce constraints on the independent parameters of the potential. Tree-level stability for large values of the fields $h$ and $S$ is ensured for positive $\lambda, b_{4}$ and $a_{2}$. However, allowing $a_{2}<0$ can enable a strong first-order EWPT [11]. In this case, requiring $4 \lambda b_{4}>a_{2}^{2}$ maintains the stability of the potential. ${ }^{4}$ This criterion becomes dependent on the cutoff of the low-energy effective theory after one takes into account the renormalization group evolution of the parameters, a consideration that we do not implement here (see, e.g. Ref., [20] and references therein). Note that for $a_{2}<0$ and/or $H$ different from Eq. (2), one may encounter additional solutions to Eq. (3) for which the $S$ vev does not vanish. We require that if such additional minima exist, the $\langle S\rangle=0$ extremum is a the global minimum. As illustrated in Fig. 1, doing so leads to the constraints in the $a_{2}-b_{3}$ plane for given values of $m_{1,2}, \theta$, and $b_{4}$. From Eq. (9) and the global stability region of Fig. 1, we then observe that for each value of $a_{2}$ there exists a minimum value of $\lambda_{211}$ consistent with the vacuum structure requirements.

\section{CURRENT CONSTRAINTS}

For the gluon-fusion-mediated process $p p \rightarrow h_{2} \rightarrow h_{1} h_{1}$ being studied here, the magnitude of the cross section depends critically on the mixing angle $\theta$ through both the $h_{2}$ coupling to SM quarks and the triscalar coupling $\lambda_{211}$. The mixing angle is constrained by the current LHC results on properties of the SM Higgs boson. On the one hand, the cross section for $p p \rightarrow h_{1}$ is reduced compared to the one for a $125 \mathrm{GeV}$ SM Higgs by a factor $c_{\theta}^{2}$ due to the singletdoublet mixing. On the other hand, although the coupling of $h_{1}$ to its decay products is also universally suppressed by $c_{\theta}$, its decay branching ratios are the same as for a SM Higgs since no new decay channels are open. Consequently, the observation of the SM-like Higgs at the LHC can be used to set a lower bound on $c_{\theta}^{2}$ due to the associated signal suppression in SM Higgs decay channels. Recent global analyses of LHC Higgs measurements then yield $c_{\theta}^{2}>0.66$ at $95 \%$ C.L. [28,29]. From the analysis in Ref. [11] we observe that for mixing angles in this range and $m_{2}>2 m_{1}$, the $\mathrm{xSM}$ can lead to a strong first-order EWPT.

Global fits to electroweak precision data also imply constraints on the mixing angle and $m_{2}$. Although a reanalysis of these constraints goes beyond the scope of the present investigation, previous studies indicate that significant singlet-doublet mixing is disfavored for heavier $h_{2}[11]$.

\footnotetext{
${ }^{4}$ A strong first-order EWPT can also occur for $a_{2} \geq 0$ for nonvanishing $a_{1}$.
} 
Another important constraint comes from ATLAS [30,31] and CMS [32] direct searches for heavy scalars decaying to $W W$ and $Z Z$. As the resulting constraints are dependent on the heavy scalar mass, we note that in the next section we will choose as benchmark scenarios for our analysis $m_{2}=270 \mathrm{GeV}$ and $m_{2}=370 \mathrm{GeV}$. ATLAS searches in the $W W$ channel exclude $h_{2}$ at 95\% C.L. for $(\sigma \times \mathrm{Br}) /(\sigma \times \mathrm{Br})_{\mathrm{SM}} \gtrsim 0.7$ for $m_{2} \sim 270 \mathrm{GeV}$ and $(\sigma \times \mathrm{Br}) /(\sigma \times \mathrm{Br})_{\mathrm{SM}} \gtrsim 0.4$ for $m_{2} \sim 370 \mathrm{GeV}$, while $\mathrm{ZZ}$ searches exclude $h_{2}$ at $95 \%$ C.L. for $(\sigma \times \mathrm{Br}) /$ $(\sigma \times \mathrm{Br})_{\mathrm{SM}} \gtrsim 0.25$ for $m_{h_{2}} \sim 270 \mathrm{GeV}$ and $(\sigma \times \mathrm{Br}) /$ $(\sigma \times \mathrm{Br})_{\mathrm{SM}} \gtrsim 0.3$ for $m_{h_{2}} \sim 370 \mathrm{GeV}$. The bounds extracted from CMS searches are found to be similar. The production cross section for $h_{2}$ in the present case is given by $s_{\theta}^{2} \sigma_{\mathrm{SM}}$, and thus for $s_{\theta}^{2} \leq 0.34$ the constraints from $W W$ searches are satisfied, while a mild reduction in the branching ratio $\operatorname{Br}\left(h_{2} \rightarrow Z Z\right)$ compared to the $\mathrm{SM}$, due to the $h_{2} \rightarrow h_{1} h_{1}$ decay channel being available, suffices to satisfy also the constraints from $Z Z$ searches.

\section{RESONANT DI-HIGGS PRODUCTION AT THE LHC}

We now consider in detail resonant di-Higgs production at the LHC for $\sqrt{s}=14 \mathrm{TeV}$. We focus on the gluonfusion production mechanism that is by far the dominant one for $m_{2}$ in the mass range of interest for the EWPT. ${ }^{5}$ The production mechanism is analogous to Higgs pair production in the SM via the trilinear Higgs self-coupling [5], except that (a) the $s$-channel $g g \rightarrow h_{2} \rightarrow h_{1} h_{1}$ amplitude may be resonant in the present case (see also Ref. [10]), and (b) the $g g h_{2}$ interaction will be reduced in strength by $c_{\theta}$.

Apart from being an important avenue for the discovery of the new heavy scalar state $h_{2}$, the process $g g \rightarrow h_{2} \rightarrow$ $h_{1} h_{1}$ is sensitive to the value of $\lambda_{211}$ when $\operatorname{Br}\left(h_{2} \rightarrow h_{1} h_{1}\right)$ is significantly smaller than 1 [in particular, for $\operatorname{Br}\left(h_{2} \rightarrow h_{1} h_{1}\right) \ll 1$, one obtains $\left.\sigma\left(g g \rightarrow h_{2} \rightarrow h_{1} h_{1}\right) \propto \lambda_{211}^{2}\right]$. For the benchmark scenarios analyzed here (see below) $m_{2}=270 \mathrm{GeV}$ and $m_{2}=370 \mathrm{GeV}$, the value of the branching ratio $\operatorname{Br}\left(h_{2} \rightarrow h_{1} h_{1}\right)$ is, respectively, 0.44 and 0.24 , and thus in both scenarios the process $g g \rightarrow h_{2} \rightarrow$ $h_{1} h_{1}$ may yield sensitivity to $\lambda_{211}$. Notice that there is an intrinsic degeneracy at this point between $\lambda_{211}$ and $c_{\theta}$, which may be disentangled via $h_{1}$ properties or by observing $h_{2}$ in other potential discovery channels, such as $h_{2} \rightarrow Z Z$.

Before discussing our rationale for focusing on the $b \bar{b} \tau^{+} \tau^{-}$final state, it is useful to compare the expected magnitudes of the resonant and nonresonant di-Higgs production cross sections for the ranges of masses and couplings we consider below. The two most important contributions to the nonresonant cross section arise from the $g g \rightarrow h_{1} h_{1}$ amplitude involving the top-quark box graph

\footnotetext{
${ }^{5}$ We defer a study of associated production, weak boson fusion, and $t \bar{t} h_{2}$ production to future work.
}

and from the $g g \rightarrow h_{1}^{*} \rightarrow h_{1} h_{1}$ process. The former will be reduced in strength from its SM value by $c_{\theta}^{2}$, while the latter will be reduced by $c_{\theta} \times \lambda_{111} / \lambda_{\mathrm{SM}}$. Taking $c_{\theta}^{2}=0.66$ and the SM di-Higgs production cross section from Ref. [5] for $\lambda_{111}=0$ we obtain $\sigma\left(p p \rightarrow h_{1} h_{1}\right)_{\text {non-res }} \approx 26 \mathrm{fb}$, which lies well below our typical values for the resonant cross section: $\mathcal{O}(1) \mathrm{pb}$ for $m_{2} \lesssim 400 \mathrm{GeV}$. Depending on the choices of the remaining independent parameters, the nonresonant $g g \rightarrow h_{1}^{*} \rightarrow h_{1} h_{1}$ process may interfere constructively with the box contribution, leading to as much as a factor of 2 increase in the total nonresonant cross section. The resulting cross section nevertheless lies well below the typical resonant production cross sections for the range of $m_{2}$ that we study here, so we may safely disregard the nonresonant $h_{1} h_{1}$ contribution in our analysis.

For the signal, we consider the $b \bar{b} \tau^{+} \tau^{-}$final state since it has a sufficiently large branching ratio to yield a significant number of events with $\sim 100 \mathrm{fb}^{-1}$ integrated luminosity yet does not contend with insurmountable backgrounds. For the final states with the largest branching ratio, $b \bar{b} b \bar{b}$ and $b \bar{b} W^{+} W^{-}$, the substantial backgrounds ( $\gtrsim 21 \mathrm{pb}$ and $\lesssim 900 \mathrm{pb}$ cross sections, respectively [5]) are challenging at best and may be insurmountable. ${ }^{6}$ In contrast, for the $b \bar{b} \tau^{+} \tau^{-}$channel the potential $\lesssim 900 \mathrm{pb} b \bar{b} W^{+} W^{-}$background gets reduced to $\lesssim 20 \mathrm{pb}$ due to the small $W \rightarrow \ell \nu$, $\tau \nu$ branching fraction, as shown in studies of this channel in the context of SM di-Higgs production. Another potentially promising search channel is the $b \bar{b} \gamma \gamma$ final state. An earlier analysis of this channel in the context of the real triplet extension of the SM [34] indicates that discovery with $\sim 100 \mathrm{fb}^{-1}$ of integrated luminosity would be possible using this final state when the triplet scalar pair-production cross section is of order 1 picobarn. As indicated above, we defer an investigation of this channel to future work.

For the simulation of resonant di-Higgs production, we include both the $g g \rightarrow h_{2} \rightarrow h_{1} h_{1}$ and $g g \rightarrow h_{2}+j \rightarrow h_{1} h_{1}+j$ processes in order to improve the reliability of the kinematic distributions of the $h_{1}$ bosons and their decay products, even though we do not explicitly make use of the presence of this additional hard jet in our analysis. For the partonic gluonfusion process, we have implemented the XSM Lagrangian together with the scalar potential (1) in FEYNRuLES [35,36], including the five-dimensional gluon-fusion effective operator $\mathcal{A}_{g} H G_{\mu \nu}^{a} G^{a \mu \nu}$ with $\mathcal{A}_{g}\left(m_{h}\right)$ receiving its leading contribution from the top-quark triangle loop. ${ }^{7}$ We stress that the use of $\mathcal{A}_{g} H G_{\mu \nu}^{a} G^{a \mu \nu}$ is perfectly justified for the study of the process $p p \rightarrow h_{2}$, since $h_{2}$ can be considered on-shell ( $m_{2} \gg \Gamma_{h_{2}}$, see below), and thus $\mathcal{A}_{g}\left(m_{h}\right)$ can be exactly

\footnotetext{
${ }^{6}$ Recent analyses of generic resonant double SM-like Higgs production in the $b \bar{b} b \bar{b}$ suggest that it might actually be possible to efficiently suppress the large $b \bar{b} b \bar{b}$ QCD background using jet-substructure techniques [33].

${ }^{7}$ We neglect the contribution from bottom loops, which yield a $<1 \%$ correction to $\mathcal{A}_{g}\left(m_{h}\right)$, below the uncertainty coming from not including beyond NLO QCD corrections.
} 
computed at leading order (LO). Signal events are generated in MADGRAPH/MADEvent 5 [37] and subsequently interfaced to PYTHIA [38] for parton showering, jet matching and hadronization using the CTEQ6L1 parton luminosities [39] set. The events are finally interfaced to PGS [40], which uses an anti- $k_{t}$ jet reconstruction algorithm. To set the overall normalization, we rescale our simulated $K$ factor $K=\sigma\left(p p \rightarrow h_{1} h_{1} X\right)_{\mathrm{NLO}} / \sigma\left(p p \rightarrow h_{1} h_{1} X\right)_{\mathrm{LO}}$ by the value computed in Ref. [41] and updated in Ref. [42] that takes into account the full set of next-to-leading-order (NLO) QCD corrections.

We perform our study for two benchmark parameterspace points:

(a) Unboosted scenario:

$m_{2}=270 \mathrm{GeV}, \quad c_{\theta}=0.812404, \quad \lambda_{211}=-325 \mathrm{GeV}$ (with $a_{2}=0, \quad b_{3}=-293 \mathrm{GeV}$ ), $\Gamma_{h_{2}}=3.36 \mathrm{GeV}$, $\operatorname{Br}\left(h_{2} \rightarrow h_{1} h_{1}\right)=0.44$.

(b) Boosted scenario:

$m_{2}=370 \mathrm{GeV}, \quad c_{\theta}=0.812404, \quad \lambda_{211}=-325 \mathrm{GeV}$ (with $a_{2}=0, \quad b_{3}=-112 \mathrm{GeV}$ ), $\Gamma_{h_{2}}=8.72 \mathrm{GeV}$, $\operatorname{Br}\left(h_{2} \rightarrow h_{1} h_{1}\right)=0.24$.

For case (a), the di-Higgs pair is produced nearly at rest in the $h_{2}$ rest frame, so the $p_{T}$ distribution for each $h_{1}$ is peaked well below $150 \mathrm{GeV}$, as illustrated in Fig. 2. There we show the $\left|\vec{p}_{T}\right|$ distribution of the $b \bar{b}$ pair produced by one of the decaying $h_{1}$ bosons along with the corresponding dominant backgrounds (see below). For this regime, the results obtained from the effective theory above are expected to agree qualitatively very well with those using the full one-loop matrix element [5]. For case (b) the diHiggs pair is boosted, with the $h_{1} p_{T}$ distribution peaking near $130 \mathrm{GeV}$ (see Fig. 2). In this regime, one approaches the limit of validity of the effective theory, so we do not consider a heavier $m_{2}$. After taking into account NLO QCD corrections as discussed above, the corresponding inclusive

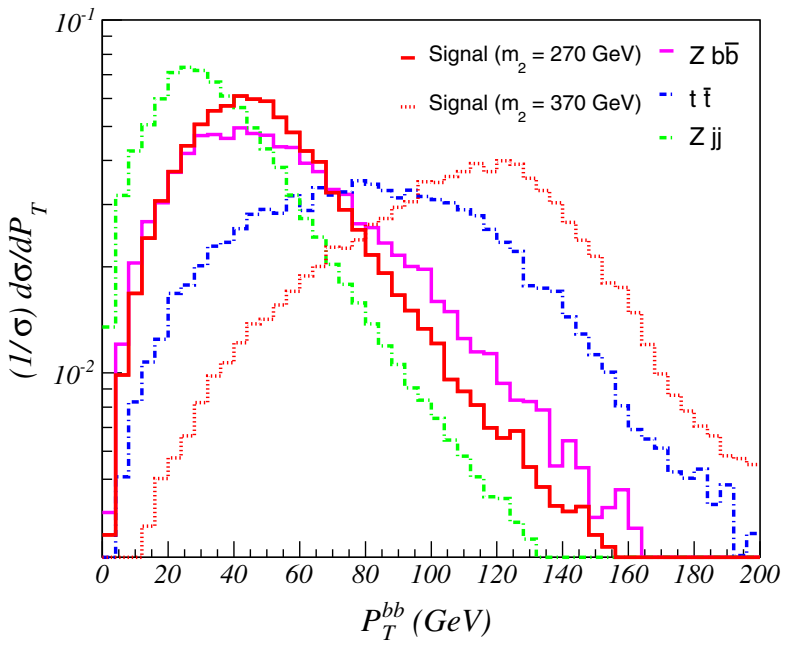

FIG. 2 (color online). Normalized $\left|\vec{P}_{T}\right|$ distribution for the $b \bar{b}$ system, for both signal and the dominant backgrounds.
di-Higgs production cross sections are $808 \mathrm{fb}(420 \mathrm{fb})$ for the unboosted (boosted) scenarios.

\section{A. Analysis of $\boldsymbol{b} \bar{b} \boldsymbol{\tau}^{+} \boldsymbol{\tau}^{-}$final states}

Maximizing the sensitivity to the $b \bar{b} \tau^{+} \tau^{-}$produced from $h_{2} \rightarrow h_{1} h_{1}$ decays entails reducing backgrounds generated by SM QCD and electroweak processes. A crucial step in this direction is the reconstruction of the invariant mass of the $b \bar{b}$ and $\tau^{+} \tau^{-}$pairs that should individually reproduce the $h_{1}$ peak. The missing mass calculator technique [43] commonly used by the ATLAS and CMS Collaborations $[44,45]$ to reconstruct the invariant mass of a $\tau^{+} \tau^{-}$system from a decaying resonance relies on maximum likelihood methods that are not possible to implement in the present analysis. Alternatively, we use the collinear approximation [46] to reconstruct the ditau invariant mass, which is used in experimental analyses of boosted resonances [45]. This procedure consists of assuming that the invisible neutrinos from the $\tau$ decays are emitted collinear with the visible products of the decay. It is then possible to obtain the absolute value of the missing momentum in each $\tau$ decay $p_{1,2}^{\text {mis }}$ using the missing energy vector $\vec{E}_{T}^{\text {miss }}$ in the event and the kinematics of the visible decay products:

$$
\begin{aligned}
& p_{1}^{\mathrm{mis}}=\frac{\sin \left(\phi_{2}^{\mathrm{vis}}\right) E_{T x}^{\mathrm{miss}}-\cos \left(\phi_{2}^{\mathrm{vis}}\right) E_{T y}^{\mathrm{miss}}}{\sin \left(\theta_{1}^{\mathrm{vis}}\right) \sin \left(\phi_{2}^{\mathrm{vis}}-\phi_{1}^{\mathrm{vis}}\right)}, \\
& p_{2}^{\mathrm{mis}}=\frac{\cos \left(\phi_{1}^{\mathrm{vis}}\right) E_{T y}^{\mathrm{miss}}-\sin \left(\phi_{1}^{\mathrm{vis}}\right) E_{T x}^{\mathrm{miss}}}{\sin \left(\theta_{2}^{\mathrm{vis}}\right) \sin \left(\phi_{2}^{\mathrm{vis}}-\phi_{1}^{\mathrm{vis}}\right)} .
\end{aligned}
$$

One then defines

$$
x_{1,2}=\frac{p_{1,2}^{\mathrm{vis}}}{p_{1,2}^{\mathrm{vis}}+p_{1,2}^{\mathrm{mis}}}
$$

where $p^{\text {vis }}$ is the absolute value of the momentum of the visible products in each $\tau$ decay. The invariant mass of the $\tau^{+} \tau^{-}$pair is then obtained as $m_{\tau \tau}^{\text {coll }}=m_{\tau \tau}^{\text {vis }} / \sqrt{x_{1} x_{2}}$, with $m_{\tau \tau}^{\text {vis }}$ being the invariant mass of the visible decay products of the $\tau^{+} \tau^{-}$system.

The primary disadvantage of the collinear approximation (10)-(12) is that it is not well defined when the two $\tau$ 's from the decay of $h$ are emitted back-to-back in the transverse plane $\left(\left|\phi_{1}-\phi_{2}\right| \sim \pi\right)$, which manifests itself in the divergence of $p_{1,2}^{\mathrm{mis}}$ as $\left|\phi_{1}-\phi_{2}\right| \rightarrow \pi$. Moreover, in this configuration, the transverse momenta of the two neutrinos will tend to cancel each other, generically resulting in little missing energy $E_{T}^{\text {miss }}$, which also renders the collinear approximation inefficient.

Imposing the collinear cut $0.1<x_{1}, x_{2}<1$ eliminates events with a back-to-back configuration, so we use it when selecting events used for the reconstruction of the ditau invariant mass, $m_{\tau \tau}^{\text {coll }}$. For the single Higgs gluon-fusion process $p p \rightarrow h \rightarrow \tau^{+} \tau^{-}$the $\tau$ leptons are generically 
emitted nearly back-to-back since the Higgs is produced almost at rest in the transverse plane. The collinear approximation is more effective for single Higgs production in conjunction with a high- $p_{T}$ jet against which the ditau pair recoils, thereby reducing the incidence of backto-back $\tau$ pairs. For di-Higgs production, the $h_{1}$ decaying to the $b \bar{b}$ pair takes the place of the high- $p_{T}$ jet, so we expect the use of the collinear approximation to be reasonably reliable in the case of our analysis (see also Ref. [45]).

The most relevant backgrounds for the analysis of $b \bar{b} \tau^{+} \tau^{-}$final states are $Z b \bar{b}, Z+$ jets (with two jets misidentified as $b$-quark objects) and $t \bar{t}$ production (the primary source of the large $b \bar{b} W^{+} W^{-}$background indicated above). As we do not consider in the present analysis the possibility of jets faking hadronically decaying $\tau$ leptons, we disregard certain possible (albeit less important) backgrounds such as $b \bar{b} W j$ and $b \bar{b} j j$. As with the signal, all background events are generated in MADGRAPH/ MadEvent 5 and subsequently interfaced to PYTHIA and PGS. The various background cross sections are normalized to their respective NLO values via enhancement $K$ factors: $K \simeq 1.4$ for $Z b \bar{b}$ [47] and $K \simeq 1.5$ for $t \bar{t}$ [48,49] (for $Z j j$, the NLO cross section is similar to the LO one for renormalization and factorization scales chosen as $\mu_{R}=$ $\mu_{F}=M_{Z}$ [50]). Following Ref. [51], our detector simulation is normalized to a $70 \% b$-tagging efficiency for $b$-quark jets with $|y|<2.5$ together with a $60 \%$ efficiency for identification of hadronic $\tau$ 's.

It is useful to organize the analysis according to the different $\tau$-decay modes, following roughly the treatment in Ref. [45]. We, thus, consider $b \bar{b}$ plus (a) two leptonically decaying $\tau$ s (" $\tau_{\text {lep }} \tau_{\text {lep }}$ "); (b) one leptonically decaying and one hadronically decaying $\tau$ (“ $\tau_{\text {lep }} \tau_{\text {had }}$ "); and (c) two hadronically decaying $\tau$ 's (" $\tau_{\text {had }} \tau_{\text {had }}$ "). After $\tau$ identification and $b$-tagging, the NLO cross sections for the unboosted (boosted) case are as follows: (a) $\tau_{\text {lep }} \tau_{\text {lep }}: 10.58(5.75) \mathrm{fb}$; (b) $\tau_{\text {lep }} \tau_{\text {had }}: 23.39(12.71) \mathrm{fb}$; and (c) $\tau_{\text {had }} \tau_{\text {had }}: 12.90(7.01) \mathrm{fb}$ for a total cross section of 46.85(25.48) fb.

\section{B. Leptonic $\left(\tau_{\mathrm{lep}} \tau_{\mathrm{lep}}\right)$ final states}

When the two $\tau$ leptons in the final state decay leptonically $\left(\tau_{\text {lep }} \tau_{\text {lep }}\right)$, the relevant backgrounds are $t \bar{t} \rightarrow b \bar{b} \ell \ell \nu \bar{\nu}$, $t \bar{t} \rightarrow b \bar{b} \ell \tau_{\text {lep }} \nu \bar{\nu}, t \bar{t} \rightarrow b \bar{b} \tau_{\text {lep }} \tau_{\text {lep }} \nu \bar{\nu}, Z b \bar{b} \rightarrow b \bar{b} \ell \ell, Z b \bar{b} \rightarrow$ $b \bar{b} \tau_{\text {lep }} \tau_{\text {lep }}, Z j j \rightarrow j j \ell \ell$ and $Z j j \rightarrow j j \tau_{\text {lep }} \tau_{\text {lep }}$. A summary of our selection and background reduction cuts for the unboosted case appears in Table I. For the boosted pair case as well as for the $\tau_{\text {lep }} \tau_{\text {had }}$ and $\tau_{\text {had }} \tau_{\text {had }}$ final states, we will subsequently discuss modifications of this basic set of cuts implemented in our analysis.

For the analysis of the $\tau_{\text {lep }} \tau_{\text {lep }}$ channel we select events containing exactly two $b$-tagged jets $\left(N_{b_{\text {tag }}}=2\right)$ and two isolated leptons $\left(N_{\ell}=2\right)$. The cuts in $\Delta R_{b b}$, the $p_{T}$ of the two $b$-tagged jets and the invariant mass reconstructions for $b \bar{b}, \tau \tau$ and $b \bar{b} \tau \tau$ significantly reduce all backgrounds (see Figs. 3,4 , and 5 ). In addition, the $Z$ backgrounds can be
TABLE I. Event selection criteria and ordered cut flow for background reduction in the $b \bar{b} \tau_{\text {lep }} \tau_{\text {lep }}$ channel.

\begin{tabular}{lc}
\hline \hline Description & Rationale \\
\hline$N_{b_{\text {tag }}}=2, N_{\ell}=2$ & signal selection \\
$p_{T}^{\ell}>10 \mathrm{GeV}$ & lepton selection \\
$p_{T}^{b}>10 \mathrm{GeV}$ & $b$-jet selection \\
$\Delta R_{b b}>0.5,\left|y_{b}\right|<2.5$ & $b$-jet selection \\
$\Delta R_{b b}>2.1$ & $Z j j, Z b \bar{b}, t \bar{t}$ reduction ${ }^{\mathrm{a}}$ \\
$P_{T, b_{1}}>45 \mathrm{GeV}, P_{T, b_{2}}>30 \mathrm{GeV}$ & $Z_{j j}, Z b \bar{b}, t \bar{t}$ reduction $^{\mathrm{b}}$ \\
$90 \mathrm{GeV}<m_{b b}<140 \mathrm{GeV}$ & $h_{1}$ mass reconstruction $^{\mathrm{c}}$ \\
$\mathrm{Collinear} x_{1}, x_{2}$ cuts & $m_{\tau \tau}^{\text {coll }}$ reconstruction \\
$\Delta R_{\ell \ell}>2$ & $t \bar{t}$ reduction ${ }^{\mathrm{d}}$ \\
$H_{T}^{\text {lept }}<120 \mathrm{GeV}$ & $t \bar{t}$ reduction \\
$30 \mathrm{GeV}<m_{\ell \ell}<75 \mathrm{GeV}$ & $Z$-peak veto \\
$30 \mathrm{GeV}<m_{e \mu}<100 \mathrm{GeV}$ & \\
$100 \mathrm{GeV}<m_{\tau \tau}^{\text {coll }}<150 \mathrm{GeV}$ & $h_{1}$ mass reconstruction \\
$E_{T}^{\text {miss }}<50 \mathrm{GeV}$ & $t \bar{t}$ reduction \\
$230 \mathrm{GeV}<m_{b b \tau \tau}^{\text {coll }}<300 \mathrm{GeV}$ & $h_{2}$ mass reconstruction \\
\hline
\end{tabular}

${ }^{\mathrm{a}}$ Fig. 3.

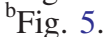

${ }^{c}$ Fig. 4.

${ }^{\mathrm{d}}$ Fig. 6 .

${ }^{\mathrm{e}}$ Fig. 7.

further suppressed by imposing cuts on the dilepton invariant mass, while $t \bar{t}$ is suppressed with a combination of cuts on $E_{T}^{\text {miss }}$, the $\Delta R$ of the reconstructed ditau pair (see Fig. 6), and the scalar sum of leptonic transverse momentum, $H_{T}^{\text {lept }}$. We include all possible combinations of opposite-sign leptons in our simulated samples $(e e, e \mu$ and $\mu \mu$ ). Further reduction of the $Z$ backgrounds could be achieved by considering only $e \mu$ pairs as in Ref. [45]. Doing so in the present case, however, leads to a loss of signal without significantly improving the final $S / \sqrt{S+B}$.

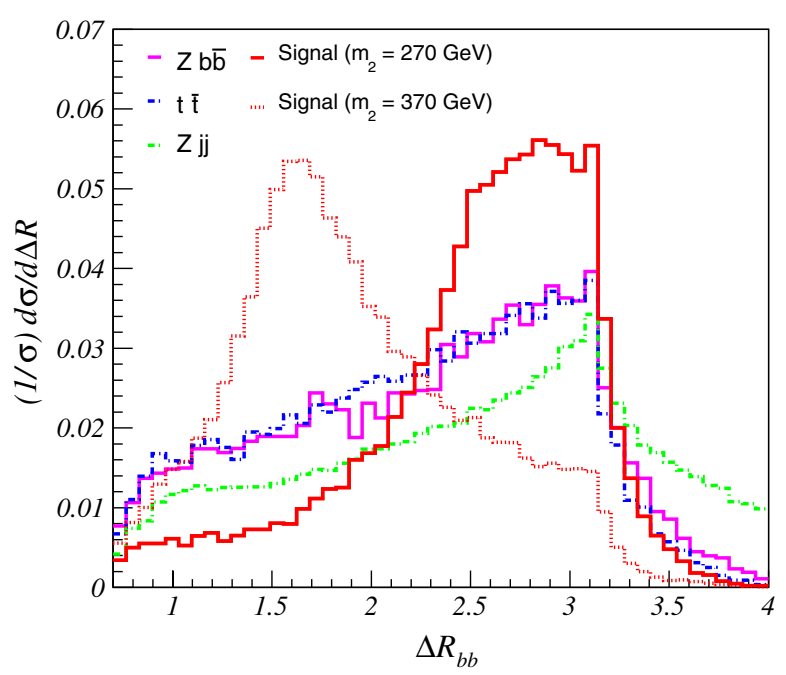

FIG. 3 (color online). Normalized $\Delta R_{b b}$ distribution after event selection (before cuts) for signal and background (" $\tau_{\text {lep }} \tau_{\text {lep }}$ "). 


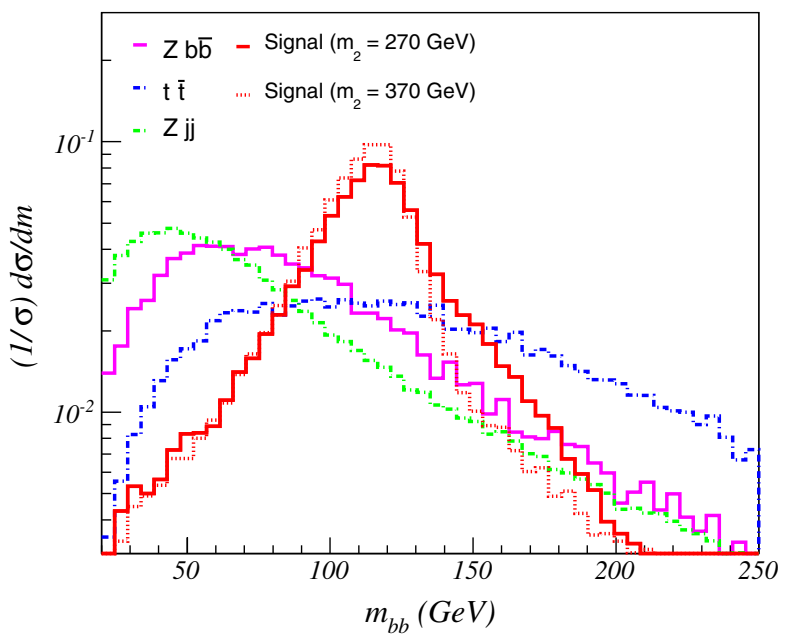

FIG. 4 (color online). Normalized $m_{b b}$ distribution after event selection (before cuts) for signal and background (" $\tau_{\text {lep }} \tau_{\text {lep }}$ ").

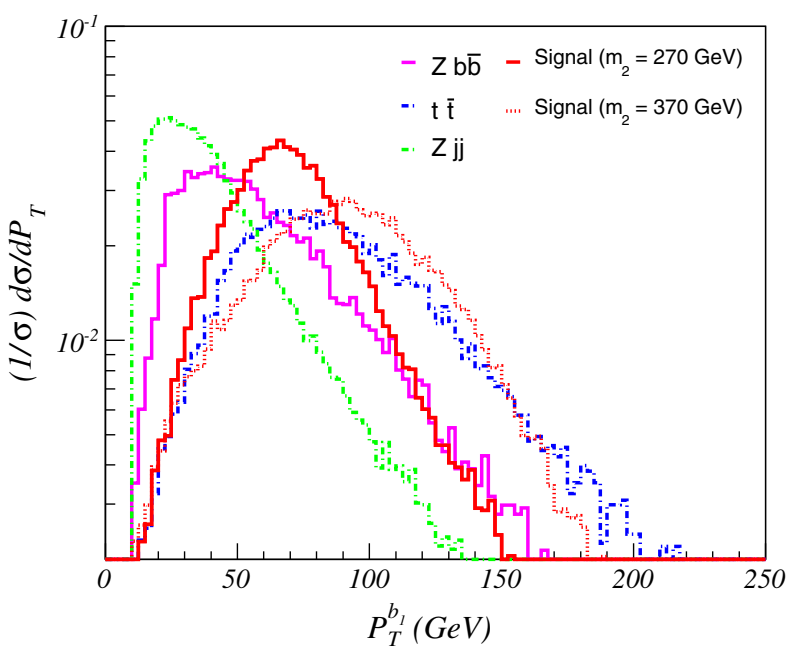

FIG. 5 (color online). Normalized $P_{T, b_{1}}$ distribution after event selection (before cuts) for signal and background (" $\tau_{\text {lep }} \tau_{\text {lep }}$ ").

For the boosted benchmark scenario, the $p_{T}$ of each $h_{1}$ will in general be substantially higher (see Fig. 2), and the $h_{1}$ decay products will tend to be more collimated. We accordingly modify our cuts by imposing an upper bound on both $\Delta R_{b b}$ and $\Delta R_{\ell \ell}$ together with an increase on the $P_{T, b_{1}}$ threshold, as suggested by Figs. 3, 5 and 6. While the $t \bar{t}$ distributions for $\Delta R_{b b}$ and $\Delta R_{\ell \ell}$ are relatively flat, those for the signal shift dramatically from the large to small $\Delta R$ range when going from the unboosted to the boosted regime (the $Z b \bar{b}$ and $Z j j$ backgrounds are reduced with separate cuts). In addition, we find further improvement in the $Z j j$ and $Z b \bar{b}$ background reduction by requiring a relatively large $\left|\vec{P}_{T}^{b b}\right|$ as is apparent from Fig. 2. The corresponding impact of the cut flow on signal and background cross sections are given in Tables II and III for the unboosted and boosted scenarios, respectively.

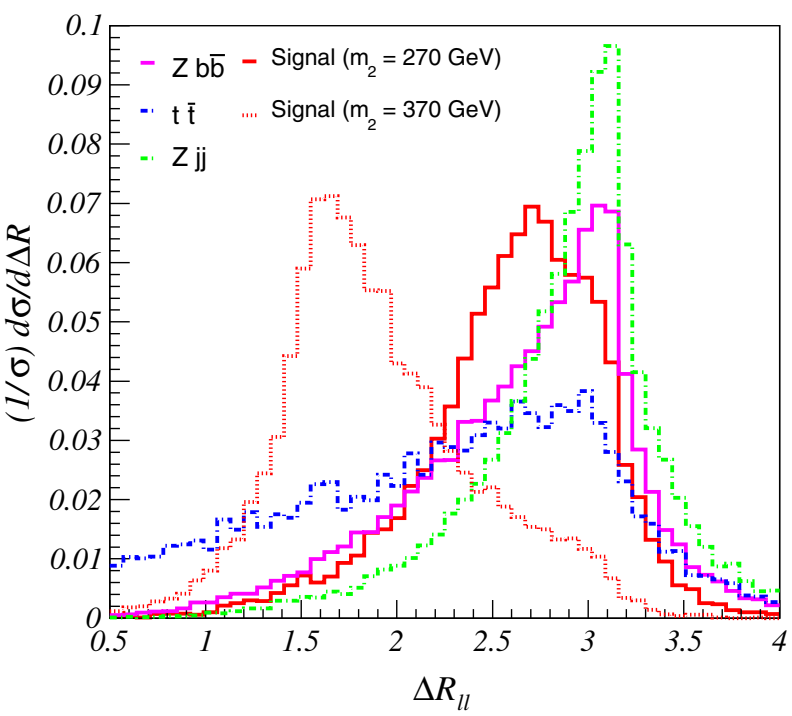

FIG. 6 (color online). Normalized $\Delta R_{\ell \ell}$ distribution after event selection (before cuts) for signal and background (" $\tau_{\text {lep }} \tau_{\text {lep }}$ ").

In light of the results from Tables II and III, for both $\tau$ leptons decaying leptonically a $S / \sqrt{S+B} \sim 5$ for the unboosted benchmark scenario can be achieved with $\sim 130-140 \mathrm{fb}^{-1}$, while the boosted benchmark scenario requires $\gtrsim 1000 \mathrm{fb}^{-1}$. The inability to efficiently reduce the $t \bar{t}$ background in the latter case is related to the greater amount of $E_{T}^{\text {miss }}$ in the signal events (coming from the decay of the more boosted $\tau$ leptons) for the boosted scenario, which then renders the cut on $E_{T}^{\text {miss }}$ relatively inefficient in suppressing the $t \bar{t}$ background, in contrast to the situation in the unboosted scenario (see Fig. 7).

\section{Semileptonic $\left(\tau_{\mathrm{lep}} \tau_{\mathrm{had}}\right)$ final states}

For the $b \bar{b} \tau_{\text {lep }} \tau_{\text {had }}$ final state, we require exactly one isolated lepton and one hadronically decaying tau (" $\tau_{h}$ "), where the latter is identified using the PGS detector simulator. The event selection criteria for this channel are given by $N_{b_{\text {tag }}}=2, N_{\ell}=1, N_{\tau_{h}}=1, p_{T}^{\ell}, p_{T}^{\tau}>10 \mathrm{GeV}$, $\left|y_{b}\right|<2.5, \Delta R_{b b}>0.5, p_{T}^{b}>10$. The main backgrounds arise from $t \bar{t}$ with $b \bar{b} \ell \tau_{\text {had }} \nu \bar{\nu}$ and $b \bar{b} \tau_{\text {lep }} \tau_{\text {had }} \nu \bar{\nu}$ produced in the $t$-quark decays, and $Z b \bar{b}, Z j j$ with $Z \rightarrow \tau_{\text {lep }} \tau_{\text {had }}$. The imposed cuts are similar to those applied to the $\tau_{\text {lep }} \tau_{\text {lep }}$ case, except for the dilepton invariant mass cuts. Instead, to reduce backgrounds associated with $b \bar{b} W W$ events (largely dominated by $t \bar{t}$ production), we cut on the transverse mass of the lepton (see Fig. 8),

$$
m_{T}^{\ell}=\sqrt{2 p_{T}^{\ell} E_{T}^{\text {miss }}\left(1-\cos \phi_{\ell, \text { miss }}\right)}<30 \mathrm{GeV},
$$

with $\phi_{\ell \text {,miss }}$ being the azimuthal angle between the direction of missing energy and the lepton transverse momentum.

The corresponding impact of the cut flow on signal and background cross sections are given in Tables IV and V for 
TABLE II. Event selection and background reduction for the $b \bar{b} \tau_{\text {lep }} \tau_{\text {lep }}$ channel in the unboosted benchmark scenario. We show the NLO cross section (in fb) for the signal $h_{2} \rightarrow h_{1} h_{1} \rightarrow b \bar{b} \tau_{\text {lep }} \tau_{\text {lep }}$ and the relevant backgrounds $t \bar{t} \rightarrow b \bar{b} \tau_{\text {lep }} \tau_{\text {lep }}, b \bar{b} \ell \tau_{\text {lep }}, b \bar{b} \ell \ell$, $Z b \bar{b} \rightarrow b \bar{b} \tau_{\text {lep }} \tau_{\text {lep }}, b \bar{b} \ell \ell$ and $Z j j \rightarrow j j \tau_{\text {lep }} \tau_{\text {lep }}, j j \ell \ell$ after successive cuts. A 70\% $b$-tagging efficiency is assumed, following Ref. [51], together with a jet fake rate of $2 \%$ (slightly more conservative than that from Ref. [51]).

\begin{tabular}{|c|c|c|c|c|c|c|}
\hline & $\begin{array}{c}h_{2} \rightarrow h_{1} h_{1} \\
b \bar{b} \tau_{\text {lep }} \tau_{\text {lep }} \\
\end{array}$ & $b \bar{b} \ell \ell$ & $\begin{array}{c}t \bar{t} \\
b \bar{b} \ell \tau_{\text {lep }}\end{array}$ & $b \bar{b} \tau_{\text {lep }} \tau_{\text {lep }}$ & $\begin{array}{c}Z b \bar{b} \\
b \bar{b} \ell \ell+b \bar{b} \tau_{\mathrm{lep}} \tau_{\text {lep }}\end{array}$ & $\begin{array}{c}Z j j \\
j j \ell \ell+j j \tau_{\text {lep }} \tau_{\text {lep }} \\
\end{array}$ \\
\hline Event selection (see Sec. V. B) & 7.47 & 11209 & 4005 & 289 & 8028 & 1144 \\
\hline$\Delta R_{b b}>2.1, P_{T, b_{1}}>45 \mathrm{GeV}, P_{T, b_{2}}>30 \mathrm{GeV}$ & 4.46 & 5585 & 2013 & 145 & 2471 & 153 \\
\hline$h_{1}$ mass: $90 \mathrm{GeV}<m_{b b}<140 \mathrm{GeV}$ & 3.12 & 1073 & 405 & 30 & 880 & 47 \\
\hline Collinear $x_{1}, x_{2}$ cuts & 2.34 & 438 & 164 & 14.1 & 248 & 18 \\
\hline$\Delta R_{\ell \ell}>2, H_{T}^{\mathrm{lept}}<120 \mathrm{GeV}$ & 2.08 & 226 & 82 & 7.9 & 200 & 16.7 \\
\hline $30 \mathrm{GeV}<m_{\ell \ell}\left(m_{e \mu}\right)<75(100) \mathrm{GeV}$ & 1.86 & 136 & 49 & 5.7 & 11.6 & 0.95 \\
\hline$h_{1}$ mass: $100 \mathrm{GeV}<m_{\tau \tau}^{\text {coll }}<150 \mathrm{GeV}$ & 1.05 & 32.5 & 11.4 & 1.63 & 3.24 & 0.24 \\
\hline$E_{T}^{\text {miss }}<50 \mathrm{GeV}$ & 0.89 & 10.5 & 3.37 & 0.56 & 3.03 & 0.23 \\
\hline$h_{2}$ mass: $230 \mathrm{GeV}<m_{b b \tau \tau}^{\text {coll }}<300 \mathrm{GeV}$ & 0.81 & 1.19 & 0.39 & 0.12 & 0.86 & 0.09 \\
\hline
\end{tabular}

TABLE III. Event selection and background reduction for the $b \bar{b} \tau_{\text {lep }} \tau_{\text {lep }}$ channel in the boosted benchmark scenario (same assumptions as in Table II).

\begin{tabular}{|c|c|c|c|c|c|c|}
\hline & $\begin{array}{c}h_{2} \rightarrow h_{1} h_{1} \\
b \bar{b} \tau_{\text {lep }} \tau_{\text {lep }} \\
\end{array}$ & $b \bar{b} \ell \ell$ & $\begin{array}{c}t \bar{t} \\
b \bar{b} \ell \tau_{\mathrm{lep}}\end{array}$ & $b \bar{b} \tau_{\text {lep }} \tau_{\text {lep }}$ & $\begin{array}{c}Z b \bar{b} \\
b \bar{b} \ell \ell+b \bar{b} \tau_{\text {lep }} \tau_{\text {lep }}\end{array}$ & $\begin{array}{c}Z j j \\
j j \ell \ell+j j \tau_{\text {lep }} \tau_{\text {lep }} \\
\end{array}$ \\
\hline Event selection (see Sec. V. B) & 4.24 & 11209 & 4005 & 289 & 8028 & 1144 \\
\hline$\Delta R_{b b}<2.2, P_{T, b_{1}}>50 \mathrm{GeV}, P_{T, b_{2}}>30 \mathrm{GeV}$ & 2.38 & 3356 & 1202 & 85 & 1166 & 35 \\
\hline$h_{1}$ mass: $90 \mathrm{GeV}<m_{b b}<140 \mathrm{GeV}$ & 1.89 & 1396 & 512 & 36 & 452 & 12 \\
\hline$\left|\vec{P}_{T}^{b b}\right|>110 \mathrm{GeV}$ & 1.35 & 719 & 264 & 19 & 208 & 4.9 \\
\hline Collinear $x_{1}, x_{2}$ cuts & 1.09 & 293 & 107 & 8.8 & 58 & 1.86 \\
\hline$\Delta R_{\ell \ell}<2.3, H_{T}^{\text {lept }}<120 \mathrm{GeV}$ & 0.80 & 120 & 45 & 4.2 & 9 & 0.14 \\
\hline $30 \mathrm{GeV}<m_{\ell \ell}\left(m_{e \mu}\right)<75(100) \mathrm{GeV}$ & 0.70 & 85 & 30 & 2.45 & 1.51 & 0.019 \\
\hline$h_{1}$ mass: $100 \mathrm{GeV}<m_{\tau \tau}^{\text {coll }}<150 \mathrm{GeV}$ & 0.60 & 30 & 11 & 0.96 & 0.24 & 0.003 \\
\hline $25 \mathrm{GeV}<E_{T}^{\text {miss }}<90 \mathrm{GeV}$ & 0.42 & 18 & 6.2 & 0.60 & 0.18 & 0.003 \\
\hline$h_{2}$ mass: $330 \mathrm{GeV}<m_{b b \tau \tau}^{\text {coll }}<400 \mathrm{GeV}$ & 0.32 & 3.25 & 1.08 & 0.11 & 0.025 & $<0.001$ \\
\hline
\end{tabular}

the unboosted and boosted scenarios. As for the $\tau_{\text {lep }} \tau_{\text {lep }}$ channel, the various cuts allow one to greatly suppress the backgrounds and increase the signal significance. For the $\tau_{\text {lep }} \tau_{\text {had }}$ channel, since it is not possible to impose a $Z$-peak

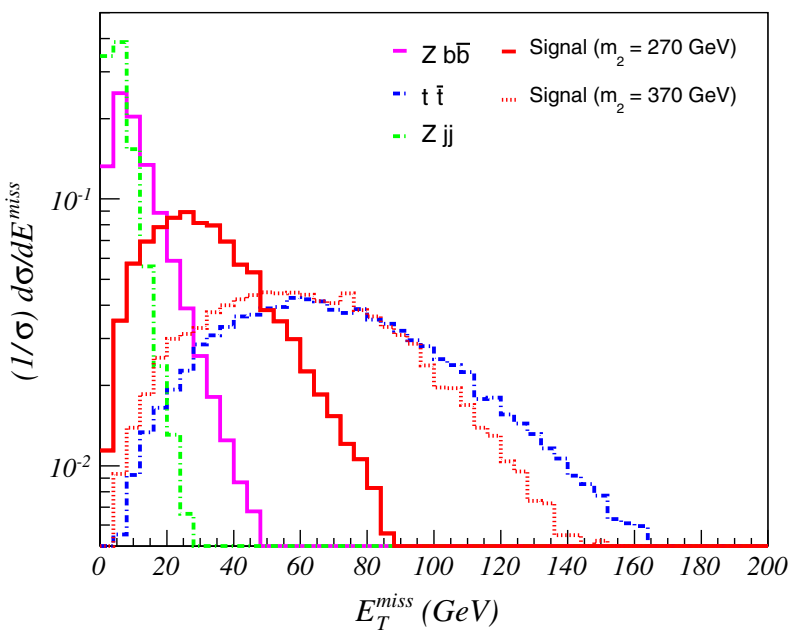

FIG. 7 (color online). Normalized $E_{T}^{\text {miss }}$ distribution after event selection (before cuts) for signal and background (" $\tau_{\text {lep }} \tau_{\text {lep }}$ ") veto through a cut in the invariant mass of the lepton pair, we increase the lower end of the $m_{\tau \tau}^{\text {coll }}$ invariant mass signal window (from $100 \mathrm{GeV}$ to $110 \mathrm{GeV}$ ) in order to suppress $Z b \bar{b}$ and $Z j j$ backgrounds. The distributions for $m_{\tau \tau}^{\text {coll }}$ and $m_{b b \tau \tau}^{\text {coll }}$ in this channel are shown in Figs. 9 and 10.

From the results from Tables IV and V, we find that for the semileptonic channel a $S / \sqrt{S+B} \sim 5$ for the unboosted benchmark scenario can be obtained with $\sim 50 \mathrm{fb}^{-1}$, while for the boosted benchmark scenario the required integrated luminosity is slightly higher, $\sim 90 \mathrm{fb}^{-1}$. This channel therefore appears to be promising both for the boosted and unboosted regimes.

\section{Hadronic ( $\left.\tau_{\text {had }} \tau_{\text {had }}\right)$ final states}

The selection criteria for this channel are given by two hadronically decaying $\tau$ leptons $\left(N_{\tau_{h}}=2\right)$, exactly zero leptons $\left(N_{\ell}=0\right)$, and a similar set of kinematic requirements on the $\tau$ leptons and $b$-jets as in the other channels: $p_{T}^{\tau}>10 \mathrm{GeV},\left|y_{b}\right|<2.5, \Delta R_{b b}>0.5, p_{T}^{b}>10$. As compared to the semileptonic and leptonic channels, the backgrounds for the purely hadronic channel are smaller. 


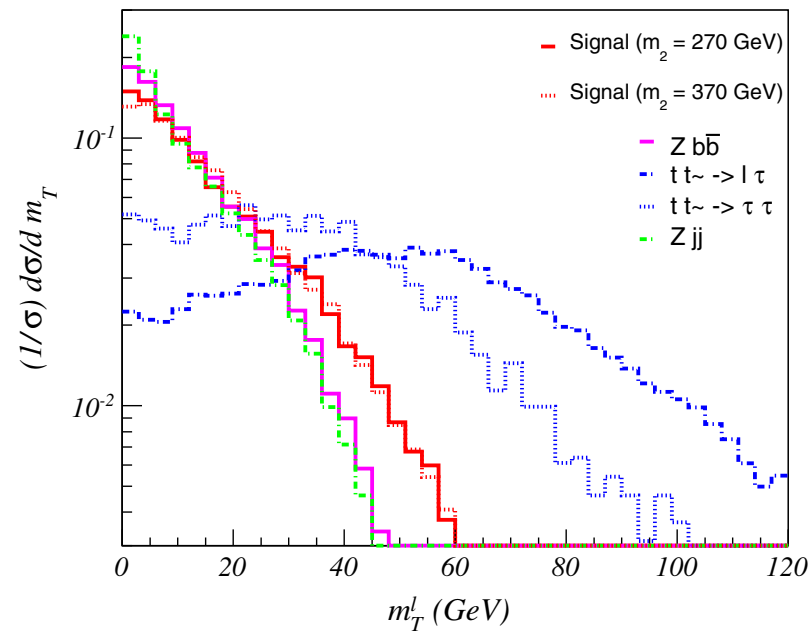

FIG. 8 (color online). Normalized $m_{T}^{\ell}$ distribution for signal and background (" $\tau_{\text {lep }} \tau_{\text {had }}$ ").

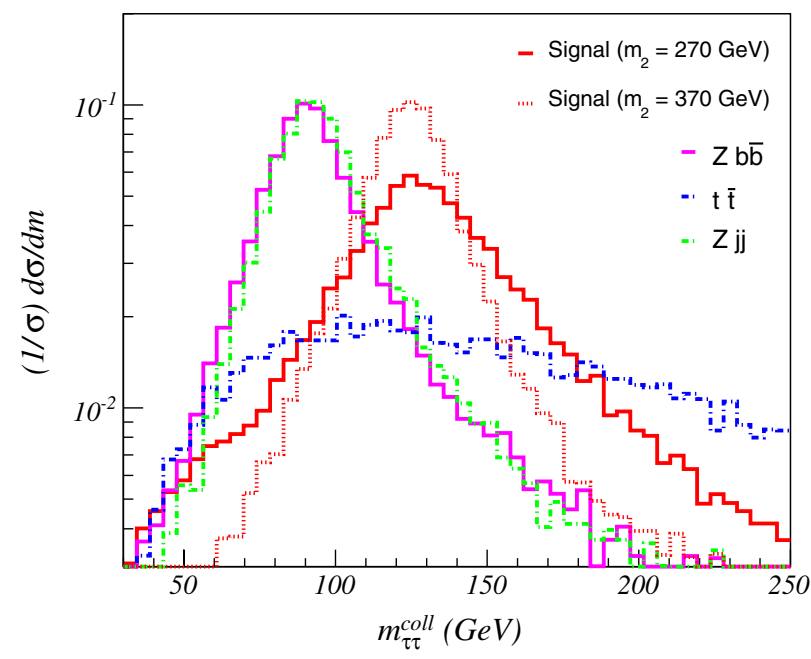

FIG. 9 (color online). Normalized $m_{\tau \tau}^{\text {coll }}$ distribution for the $\tau^{+} \tau^{-}$system in signal and background (" $\tau_{\text {lep }} \tau_{\text {had }}$ ").

The cut flows for the unboosted and boosted scenarios are given in Tables VI and VII, respectively.

In light of the results from Tables VI and VII, we obtain $S / \sqrt{S+B} \sim 5$ with $\sim 100 \mathrm{fb}^{-1}$ in the hadronic channel for both the unboosted and boosted benchmark scenarios. While this channel appears to be promising for both scenarios, we caution that we have not considered other pure QCD backgrounds, such as multijet or $b \bar{b} j j$ production, where the jets fake a hadronically decaying $\tau$ lepton. The reason is the difficulty of reliably quantifying the jet fake rate for these events, which while being under 5\%, depends strongly on the characteristics of the jet [51]. While we do not expect this class of background contamination to be an impediment to signal observation in the $\tau_{\text {had }} \tau_{\text {had }}$ channel, we are less confident

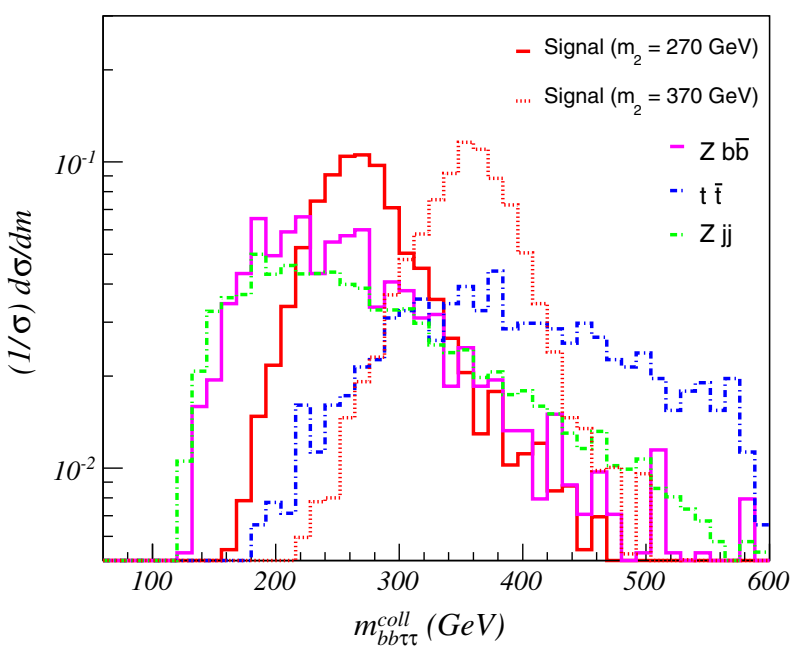

FIG. 10 (color online). Normalized $m_{b b \tau \tau}^{\text {coll }}$ distribution for signal and background (" $\tau_{\text {lep }} \tau_{\text {had }}$ ").

in our quantitative statements here than for the other final states.

\section{DISCUSSION AND OUTLOOK}

Uncovering the full structure of the SM scalar sector and its possible extensions will be a central task for the LHC in the coming years. The results will have important implications not only for our understanding of the mechanism of electroweak symmetry breaking but also for the origin of visible matter and the nature of dark matter. Extensions of the SM scalar sector that address one or both of these open questions may yield distinctive signatures at the LHC associated with either modifications of the SM Higgs boson properties and/or the existence of new states.

In this study, we have considered one class of Higgs portal scalar sector extensions containing a singlet scalar that can mix with the neutral component of the $\mathrm{SU}(2)_{L}$ doublet leading to two neutral states $h_{1,2}$. This xSM scenario can give rise to a strong first-order electroweak phase transition as needed for electroweak baryogenesis; it maps directly onto the NMSSM in the decoupling limit and it serves as a simple paradigm for mixed-state signatures in Higgs portal scenarios that contain other $\mathrm{SU}(2)_{L}$ representations. Considering resonant di-Higgs production $p p \rightarrow h_{2} \rightarrow h_{1} h_{1}$, we have shown that a search for the $b \bar{b} \tau^{+} \tau^{-}$final state could lead to the discovery of this scenario with $\sim 100 \mathrm{fb}^{-1}$ integrated luminosity for regions of the model parameter space of interest to cosmology. The most promising mode appears to involve one leptonically decaying and one hadronically decaying $\tau$ lepton, though for $m_{2}$ close to $2 m_{1}$ the purely leptonic decay modes of the $\tau$ 's could also yield discovery as well. For purely hadronically decaying $\tau$ leptons, the significance obtained from our analysis looks promising, though a more refined study of 
TABLE IV. Event selection and background reduction for the $b \bar{b} \tau_{\text {lep }} \tau_{\text {had }}$ channel in the unboosted benchmark scenario. We show the NLO cross section (in fb) for the signal $h_{2} \rightarrow h_{1} h_{1} \rightarrow b \bar{b} \tau_{\text {lep }} \tau_{\text {had }}$ and the relevant backgrounds $t \bar{t} \rightarrow b \bar{b} \tau_{\text {lep }} \tau_{\text {had }}, b \bar{b} \ell \tau_{\text {had }}$, $Z b \bar{b} \rightarrow b \bar{b} \tau_{\text {lep }} \tau_{\text {had }}$ and $Z j j \rightarrow j j \tau_{\text {lep }} \tau_{\text {had }}$ after successive cuts (same efficiency and fake rate assumptions as in Table II).

\begin{tabular}{|c|c|c|c|c|c|}
\hline & $\begin{array}{c}h_{2} \rightarrow h_{1} h_{1} \\
b \bar{b} \tau_{\text {lep }} \tau_{\text {had }}\end{array}$ & $b \bar{b} \ell \tau_{\mathrm{had}}$ & $b \bar{b} \tau_{\text {lep }} \tau_{\text {had }}$ & $\begin{array}{c}Z b \bar{b} \\
b \bar{b} \tau_{\text {lep }} \tau_{\text {had }}\end{array}$ & $\begin{array}{c}Z j j \\
j j \tau_{\text {lep }} \tau_{\text {had }} \\
\end{array}$ \\
\hline Event selection (see Sec. V.C) & 19.17 & 5249 & 762 & 601 & 98 \\
\hline$\Delta R_{b b}>2.1, P_{T, b_{1}}>45 \mathrm{GeV}, P_{T, b_{2}}>30 \mathrm{GeV}$ & 11.45 & 2639 & 384 & 188 & 10.8 \\
\hline$h_{1}$ mass: $90 \mathrm{GeV}<m_{b b}<140 \mathrm{GeV}$ & 8.00 & 531 & 80 & 69 & 3.68 \\
\hline Collinear $x_{1}, x_{2}$ cuts & 4.81 & 209 & 36.4 & 41.6 & 2.41 \\
\hline$\Delta R_{\ell \tau}>2$ & 4.10 & 129 & 23.1 & 26.5 & 2.03 \\
\hline$m_{T}^{\ell}<30 \mathrm{GeV}$ & 3.44 & 30.9 & 11.1 & 24.4 & 1.90 \\
\hline$h_{1}$ mass: $110 \mathrm{GeV}<m_{\tau \tau}^{\text {coll }}<150 \mathrm{GeV}$ & 1.56 & 4.97 & 2.05 & 4.92 & 0.38 \\
\hline$E_{T}^{\text {miss }}<50 \mathrm{GeV}$ & 1.37 & 3.31 & 0.87 & 4.29 & 0.36 \\
\hline$h_{2}$ mass: $230 \mathrm{GeV}<m_{b b \tau \tau}^{\text {coll }}<300 \mathrm{GeV}$ & 1.29 & 0.39 & 0.17 & 1.21 & 0.13 \\
\hline
\end{tabular}

TABLE V. Event selection and background reduction for the $b \bar{b} \tau_{\text {lep }} \tau_{\text {had }}$ channel in the boosted benchmark scenario (same efficiency and fake rate assumptions as in Table II).

\begin{tabular}{|c|c|c|c|c|c|}
\hline & $\begin{array}{c}h_{2} \rightarrow h_{1} h_{1} \\
b \bar{b} \tau_{\text {lep }} \tau_{\text {had }}\end{array}$ & $b \bar{b} \ell \tau_{\mathrm{had}}$ & $b \bar{b} \tau_{\text {lep }} \tau_{\text {had }}$ & $\begin{array}{c}Z b \bar{b} \\
b \bar{b} \tau_{\text {lep }} \tau_{\text {had }}\end{array}$ & $\begin{array}{c}Z j j \\
j j \tau_{\text {lep }} \tau_{\text {had }} \\
\end{array}$ \\
\hline Event selection (see Sec. V.C) & 10.73 & 5249 & 762 & 601 & 98 \\
\hline$\Delta R_{b b}<2.2, P_{T, b_{1}}>50 \mathrm{GeV}, P_{T, b_{2}}>30 \mathrm{GeV}$ & 6.02 & 1576 & 223 & 85 & 2.46 \\
\hline$h_{1}$ mass: $90 \mathrm{GeV}<m_{b b}<140 \mathrm{GeV}$ & 4.77 & 672 & 94 & 31.5 & 0.84 \\
\hline$\left|\vec{P}_{T}^{b b}\right|>110 \mathrm{GeV}$ & 3.42 & 345 & 49 & 13.9 & 0.33 \\
\hline Collinear $x_{1}, x_{2}$ cuts & 2.31 & 136 & 22.3 & 8.38 & 0.22 \\
\hline$\Delta R_{\ell \tau}<2.3$ & 1.71 & 68 & 11.1 & 4.31 & 0.055 \\
\hline$m_{T}^{\ell}<30 \mathrm{GeV}$ & 1.46 & 18.4 & 5.64 & 4.02 & 0.051 \\
\hline$h_{1}$ mass: $110 \mathrm{GeV}<m_{\tau \tau}^{\text {coll }}<150 \mathrm{GeV}$ & 1.05 & 4.2 & 1.26 & 0.30 & 0.003 \\
\hline $25 \mathrm{GeV}<E_{T}^{\text {miss }}<90 \mathrm{GeV}$ & 0.76 & 2.93 & 0.75 & 0.23 & 0.002 \\
\hline$h_{2}$ mass: $330 \mathrm{GeV}<m_{b b \tau \tau}^{\text {coll }}<400 \mathrm{GeV}$ & 0.63 & 0.60 & 0.15 & 0.026 & $<0.001$ \\
\hline
\end{tabular}

TABLE VI. Event selection and background reduction for the $b \bar{b} \tau_{\text {had }} \tau_{\text {had }}$ channel in the unboosted benchmark scenario. We show the NLO cross section (in fb) for the signal $h_{2} \rightarrow h_{1} h_{1} \rightarrow b \bar{b} \tau_{\text {had }} \tau_{\text {had }}$ and the relevant backgrounds $t \bar{t} \rightarrow b \bar{b} \tau_{\text {had }} \tau_{\text {had }}, Z b \bar{b} \rightarrow b \bar{b} \tau_{\text {had }} \tau_{\text {had }}$ and $Z j j \rightarrow j j \tau_{\text {had }} \tau_{\text {had }}$ after successive cuts (same efficiency and fake rate assumptions as in Table II).

\begin{tabular}{|c|c|c|c|c|}
\hline & $\begin{array}{c}h_{2} \rightarrow h_{1} h_{1} \\
b \bar{b} \tau_{\text {had }} \tau_{\text {had }} \\
\end{array}$ & $b \bar{b} \tau_{\mathrm{had}} \tau_{\mathrm{had}}$ & $\begin{array}{c}Z b \bar{b} \\
b \bar{b} \tau_{\text {had }} \tau_{\text {had }} \\
\end{array}$ & $\begin{array}{c}Z j j \\
j j \tau_{\text {had }} \tau_{\text {had }} \\
\end{array}$ \\
\hline Event selection (see Sec. V.D) & 12.31 & 509 & 411 & 67 \\
\hline$\Delta R_{b b}>2.1, P_{T, b_{1}}>45 \mathrm{GeV}, P_{T, b_{2}}>30 \mathrm{GeV}$ & 7.35 & 256 & 128 & 7.39 \\
\hline$h_{1}$ mass: $90 \mathrm{GeV}<m_{b b}<140 \mathrm{GeV}$ & 5.14 & 53 & 47 & 2.52 \\
\hline Collinear $x_{1}, x_{2}$ cuts & 2.57 & 22.8 & 24.5 & 1.42 \\
\hline$\Delta R_{\tau \tau}>2$ & 2.04 & 12.4 & 15.8 & 1.19 \\
\hline$h_{1}$ mass: $110 \mathrm{GeV}<m_{\tau \tau}^{\text {coll }}<150 \mathrm{GeV}$ & 0.82 & 1.79 & 3.75 & 0.27 \\
\hline$E_{T}^{\mathrm{miss}}<50 \mathrm{GeV}$ & 0.75 & 0.60 & 3.39 & 0.26 \\
\hline$h_{2}$ mass: $230 \mathrm{GeV}<m_{b b \tau \tau}^{\text {coll }}<300 \mathrm{GeV}$ & 0.72 & 0.08 & 1.03 & 0.11 \\
\hline
\end{tabular}

the rate for jets faking hadronically decaying $\tau$ 's would give one more confidence in the prospects for this mode.

The study of other final states formed from combinations of SM Higgs decay products, as suggested by the work of Ref. [15] that appeared as we were completing this paper, would be a natural next step. Although we disagree with the quantitative results in that study (a preliminary application of their basic cuts to the $b \bar{b} \tau^{+} \tau^{-}$final state yields $S / B \sim 1$ rather than the $\sim 200$ as these authors find), we concur that a detailed analysis of other novel states associated 
TABLE VII. Event selection and background reduction for the $b \bar{b} \tau_{\text {had }} \tau_{\text {had }}$ channel in the boosted benchmark scenario (same efficiency and fake rate assumptions as in Table II).

\begin{tabular}{lccc}
\hline \hline & $\begin{array}{c}h_{2} \rightarrow h_{1} h_{1} \\
b \bar{b} \tau_{\text {had }} \tau_{\text {had }}\end{array}$ & $\begin{array}{c}Z \bar{t} \\
b \bar{b} \tau_{\text {had }} \tau_{\text {had }}\end{array}$ & $\begin{array}{c}Z b \bar{b} \\
b \bar{b} \tau_{\text {had }} \tau_{\text {had }}\end{array}$ \\
\hline Event selection (see Sec. V.D) & 6.71 & 411 \\
$\Delta R_{b b}<2.2, P_{T, b_{1}}>50 \mathrm{GeV}, P_{T, b_{2}}>30 \mathrm{GeV}$ & 309 & 58 & 67 \\
$h_{1}$ mass: $90 \mathrm{GeV}<m_{b b}<140 \mathrm{GeV}$ & 3.77 & 149 & 1.68 \\
$\left|\vec{P}_{T}^{b b}\right|>110 \mathrm{GeV}$ & 2.99 & 63 & 0.57 \\
Collinear $x_{1}, x_{2}$ cuts & 2.14 & 32.5 & 9.5 \\
$\Delta R_{\tau \tau}<2.3$ & 1.27 & 13.9 & 4.95 \\
$h_{1}$ mass: $110 \mathrm{GeV}<m_{\tau \tau}^{\text {coll }}<150 \mathrm{GeV}$ & 0.92 & 8.1 & 2.51 \\
$25 \mathrm{GeV}<E_{T}^{\text {miss }}<90 \mathrm{GeV}$ & 0.64 & 1.91 & 0.23 \\
$h_{2}$ mass: $330 \mathrm{GeV}<m_{b b \tau \tau}^{\text {coll }}<400 \mathrm{GeV}$ & 0.47 & 0.98 & 0.13 \\
\hline \hline
\end{tabular}

with resonant di-Higgs production would be a worthwhile effort.

\section{ACKNOWLEDGMENTS}

J. M. N. thanks Veronica Sanz for very useful discussions. M. J. R. M. thanks B. Brau, C. Dallapiccola, and S. Willocq for helpful discussions and comments on the manuscript. Both authors thank H. Guo, T. Peng, and H. Patel for generating background event samples. J. M. N. is supported by the Science Technology and Facilities Council (STFC) under grant No. ST/J000477/1. M. J. R. M. was supported in part by U.S. Department of Energy Contract No. DE-FG02-08ER41531 and the Wisconsin Alumni
Research Foundation. The authors also thank the Excellence Cluster Universe at the Technical University of Munich, where a portion of this work was carried out.

Note added.-Recently, the authors of Ref. [15] clarified that the source of discrepancy with our results is ultimately related to the difference in the production cross section $\sigma\left(p p \rightarrow h_{2}\right)$ between us and them. It can be seen (by comparing the $h_{2}$ production cross section to the corresponding production cross sections of a SM Higgs of the same mass) that the values of $\sigma\left(p p \rightarrow h_{2}\right)$ quoted in Ref. [15] (e.g. $~ 30$ pb for $m_{2}=300 \mathrm{GeV}$ ) are not possible to obtain in general.
[1] A. Djouadi, W. Kilian, M. Muhlleitner, and P. Zerwas, Eur. Phys. J. C 10, 45 (1999).

[2] U. Baur, T. Plehn, and D. L. Rainwater, Phys. Rev. D 67, 033003 (2003).

[3] U. Baur, T. Plehn, and D. L. Rainwater, Phys. Rev. D 68, 033001 (2003).

[4] U. Baur, T. Plehn, and D. L. Rainwater, Phys. Rev. D 69, 053004 (2004).

[5] M. J. Dolan, C. Englert, and M. Spannowsky, J. High Energy Phys. 10 (2012) 112.

[6] A. Papaefstathiou, L. L. Yang, and J. Zurita, Phys. Rev. D 87, 011301 (2013).

[7] F. Goertz, A. Papaefstathiou, L. L. Yang, and J. Zurita, J. High Energy Phys. 06 (2013) 016.

[8] A. J. Barr, M. J. Dolan, C. Englert, and M. Spannowsky, Phys. Lett. B 728, 308 (2014).

[9] D. E. Morrissey and M. J. Ramsey-Musolf, New J. Phys. 14, 125003 (2012).

[10] M. J. Dolan, C. Englert, and M. Spannowsky, Phys. Rev. D 87, 055002 (2013).

[11] S. Profumo, M. J. Ramsey-Musolf, and G. Shaughnessy, J. High Energy Phys. 08 (2007) 010.
[12] J. R. Espinosa, T. Konstandin, and F. Riva, Nucl. Phys. B854, 592 (2012).

[13] M. Maniatis, Int. J. Mod. Phys. A 25, 3505 (2010).

[14] U. Ellwanger, C. Hugonie, and A. M. Teixeira, Phys. Rep. 496, 1 (2010).

[15] J. Liu, X.-P. Wang, and S.-h. Zhu, arXiv:1310.3634.

[16] D. O'Connell, M. J. Ramsey-Musolf, and M. B. Wise, Phys. Rev. D 75, 037701 (2007).

[17] J. McDonald, Phys. Rev. D 50, 3637 (1994).

[18] C. Burgess, M. Pospelov, and T. ter Veldhuis, Nucl. Phys. B619, 709 (2001).

[19] V. Barger, P. Langacker, M. McCaskey, M. Ramsey-Musolf, and G. Shaughnessy, Phys. Rev. D 79, 015018 (2009).

[20] M. Gonderinger, H. Lim, and M. J. Ramsey-Musolf, Phys. Rev. D 86, 043511 (2012).

[21] J. R. Espinosa, C. Grojean, V. Sanz, and M. Trott, J. High Energy Phys. 12 (2012) 077.

[22] R. Barbieri, D. Buttazzo, K. Kannike, F. Sala, and A. Tesi, Phys. Rev. D 87, 115018 (2013).

[23] G. Belanger, B. Dumont, U. Ellwanger, J. Gunion, and S. Kraml, Phys. Rev. D 88, 075008 (2013).

[24] U. Ellwanger, J. High Energy Phys. 08 (2013) 077. 
[25] J. Cao, Z. Heng, L. Shang, P. Wan, and J. M. Yang, J. High Energy Phys. 04 (2013) 134.

[26] Z. Kang, J. Li, T. Li, D. Liu, and J. Shu, Phys. Rev. D 88, 015006 (2013).

[27] V. Barger, P. Langacker, M. McCaskey, M. J. RamseyMusolf, and G. Shaughnessy, Phys. Rev. D 77, 035005 (2008).

[28] P. Winslow (private communication).

[29] ATLAS Collaboration, Report No. ATLAS-Conf-2013-034.

[30] ATLAS Collaboration, Report No. ATLAS-Conf-2013-067.

[31] ATLAS Collaboration, Report No. ATLAS-Conf-2013-013.

[32] CMS Collaboration, Eur. Phys. J. C 73, 2469 (2013).

[33] M. Gouzevitch, A. Oliveira, J. Rojo, R. Rosenfeld, G. P. Salam, and V. Sanz, J. High Energy Phys. 07 (2013) 148.

[34] P. Fileviez Perez, H. H. Patel, M. Ramsey-Musolf, and K. Wang, Phys. Rev. D 79, 055024 (2009).

[35] N. D. Christensen and C. Duhr, Comput. Phys. Commun. 180, 1614 (2009).

[36] C. Degrande, C. Duhr, B. Fuks, D. Grellscheid, O. Mattelaer, and T. Reiter, Comput. Phys. Commun. 183, 1201 (2012).

[37] J. Alwall, M. Herquet, F. Maltoni, O. Mattelaer, and T. Stelzer, J. High Energy Phys. 06 (2011) 128.

[38] T. Sjostrand, S. Mrenna, and P. Z. Skands, Comput. Phys. Commun. 178, 852 (2008).

[39] J. Pumplin, D. Robert Stump, J. Huston, H.-L. Lai, P. Nadolsky, and W.-K. Tung, J. High Energy Phys. 07 (2002) 012.
[40] http://www.physics.ucdavis.edu/ conway/research/software/ pgs/pgs4-general.htm.

[41] S. Dawson, S. Dittmaier, and M. Spira, Phys. Rev. D 58, 115012 (1998).

[42] J. Baglio, A. Djouadi, R. Gröber, M.M. Mühlleitner, J. Quevillon, and M. Spira, J. High Energy Phys. 04 (2013) 151.

[43] A. Elagin, P. Murat, A. Pranko, and A. Safonov, Nucl. Instrum. Methods Phys. Res., Sect. A 654, 481 (2011).

[44] S. Chatrchyan et al. (CMS Collaboration), Phys. Lett. B 713, 68 (2012).

[45] G. Aad et al. (ATLAS Collaboration), J. High Energy Phys. 09 (2012) 070.

[46] R. K. Ellis, I. Hinchliffe, M. Soldate, and J. van der Bij, Nucl. Phys. B297, 221 (1988).

[47] J. M. Campbell, R. K. Ellis, F. Maltoni, and S. Willenbrock, Phys. Rev. D 73, 054007 (2006).

[48] M. L. Mangano, P. Nason, and G. Ridolfi, Nucl. Phys. B373, 295 (1992).

[49] G. Bevilacqua, M. Czakon, A. van Hameren, C. G. Papadopoulos, and M. Worek, J. High Energy Phys. 02 (2011) 083.

[50] J. M. Campbell, R. K. Ellis, and D. L. Rainwater, Phys. Rev. D 68, 094021 (2003).

[51] ATLAS Collaboration, Tech. Rept. ATL-PHYS-PUB-2013004. 